\title{
The Various Viewpoints toward a Language Time and Aspect Based on the aspects of English and Korean Language
}

\author{
Ji-Hye Lee ${ }^{1}$ and Mun-Koo Kang ${ }^{2 *}$ \\ ${ }^{1}$ Department of English Education, Kongju National University, \\ 56 Gongjudaehak-ro, Gongju-si, Chungcheongnam-do, 314-701, Republic of Korea \\ $2^{*}$ Department of English Education, Kongju National University, \\ 56 Gongjudaehak-ro, Gongju-si, Chungcheongnam-do, 314-701, Republic of Korea \\ 1yjh2488@naver.com, ${ }^{2 *}$ kangmunkoo@hanmail.net
}

\begin{abstract}
The tense presented in a verb has general traits, expressing time. But an aspect has a different trait and a special case according to each language. Take a look at how different the aspects indicating how to see a situation are in the English and Korean language. Also, look at how the concept of aspect can be identified in the two languages. In order to understand a grammatical concept which expresses an aspect that does not exist in Korean language, it is essential for learners to know the tools and mediums which can help clarify the meaning of aspect and figure out the characteristics of standard aspects.
\end{abstract}

Keywords: Aspect, Perfective, Perfect, Viewing situation, A figure of speech

\section{The origin of aspect and the meaning}

Both the concept of direct time that people recognize and the thought of a different time meaning other than what its represents in the relation were already discovered. Among these discoveries, we can see the concept of "aspect" in Varro's studies. The use of "aspect" in a modern society, however, was discovered in the N.I.Grech's Russian grammar which was first published in 1827. He used a Russian word, 'vid' and translated it into 'aspect' in French. Like all Slavish language, most verbs in Russian language have two different forms in a morphological way. These forms present the two ways of viewing a situation, which are 'perspective' and 'imperfective' People use different terms when it comes to expressing an aspect and some scientists have a more specific distinction. But the distinction of 'perspective' and 'imperfective' belongs to our basic frame. For example, Comrie (1976) distinguishes 'imperfective' from 'habitual' and 'continuous'. The latter divides into 'nonprogressive' and 'progressive'.

\section{The traits of standard aspect}

\subsection{The viewing of the situation}

There is no doubt that language is a medium to present the time and place of a speaker and listener. A distinctive example of this is a deixis. That's because it can be 'left' or 'right'

Article history:

Received (September 19, 2016), Review Result (November 05, 2016), Accepted (December 09, 2016) 
according to the speaker's position even they are in an identified relation. In some point of view, deixis-relative tense can be connected with the time and place. But all scientists emphasize that aspect is not a category of deixis, unlike a tense. Thus, an aspect doesn't reflect this kind of 'subjectivity'. (Lyons 1982)

\subsection{Completion}

'Completed' has two views like the following.

The concept of time completion is relative when it comes to a 'time'. This does not mean that a time should be identified explicitly, but it always implies the meaning.

Like what Comrie mentioned before, the concept of 'completion' focuses on the last moment of each situation too much.

\subsection{Figure of speech for space}

What does the expressions, 'from inside' and 'from outside' in a concept of space mean? There is no doubt that these two figure of speech connote an important part about an aspect. It is difficult describing this correctly. Then, what is a boundary of aspect? Relating to this question, Comrie suggests that the distinction of basic aspect does not use a confrontation of the definitions for 'inner' and 'outer' in a direct way. Though Klein accepted this argument from Comie, he also pointed out the following limitations from the argument. Firstly, this is a contemplative figure of speech. Secondly, and more importantly, 'inner construction' allows a difference of aspect or it is not clear that what it means in an apathetic situation which is at least allowed very often. This is persuasive, but it is not a satisfactory figure of speech and too metaphorical rather than a distinctive definition.

\section{The aspect concept of English}

\subsection{The content of 0 - situation}

According to Klein's definition of aspect, the contents of 0-state do not show any contrasts or topic time. If the content can be connected in a certain topic time, this will be connected automatically in a different topic time.

\subsection{The 1 for vocabulary content of situation}

This means all situations which embrace prior time and post time on a situation time basis. But the situation time does not mean a situation without a beginning and ending. The situation time just includes a part of topic time. This time, the situation time and a topic time can be overlapped or the situation time is included in a topic time.

\subsection{The 2 for vocabulary content of situation}

This includes the initial state and a goal state. Thus, these allow more complex and possible relationship than the two factors which mentioned before. Topic time can be placed in an initial state, a goal state, a part of these two states, or a prior state. 


\subsection{Post time: Perfective and perfect}

Post time can make a time-based definition. This time refers to a post time after a situation time. A situation time itself can be inferred from a topic time or if it is possible, it is inferred from a certain aspect front cover and vocabulary content itself in a case of 0 -situation. A concept of post time plays an important role in the definition of both perfective and perfect. In perfective, topic time is partially included in a post time. A topic time in perfect, situates completely far from a post time. Take a look at the following examples.

a) John slept (perfective)

b) John had slept (perfect)

In a survey research about an aspect, both perfective and perfect are related with a completion of situation or a concept of completion. However, if the completion does not make a connection with a time like 'when did it complete?', there would be no meaning. As I mentioned before, a steamed egg means an egg which steaming finishes completion. But this completion is connected with a specific time which does not complete before. It is not essential to mention this time but it exists implicitly.

\subsection{The types of the perfect}

Many languages have inflectional endings or used verb forms similar to it, which are called perfect. In this chapter, I am going to examine the completion of English and various interpretations which are given in the perfect. The following information comes from documented researches.

There have been an extensive studies regarding to the perfect of English. Here the main problem is whether the unique meaning of the perfect form really exists or not when the various usages are given. Klein (1994) believes that it really exists, which means topic time is included in a period after situation time. But he did not mention about the distance between topic time and situation time.

The following are types of the perfect which have appeared on documented researches.

The Consequential perfect: The present in consequential perfect is indicated as a certain outcome of the past situation. For example, the possible answer to these questions is, 'Is John still here?' and 'Hasn't John comes yet?' is not 'Yes, he arrived.' but 'Yes, he has arrived.'

The Experiential perfect: This means that a given situation occurs at least one time and continues to maintain from a moment of the past to the present. For example, the sentence, 'Bill has been to America.' means that he visited USA at least once, by contrast with 'Bill has gone to America.' In the latter, the distance between topic time and situation time can be longer at the extent of not being recognized. Especially, topic time does not have to be in the proximal part of time if the proximal part of the post time is distinguished from a following part.

The perfect of continual situation: The usage of the perfect typically shown in English is that the beginning happened in the past, as seen in 'We have lived here for ten years.' but it describes the situation which continues until the present. Klein views it as a subtle thing. The reason why the situation time in the perfect like 'We have lived here for ten years.' includes a topic time, is that this topic time seems to include an utterance time. When considering the contents of overall describing vocabularies, the situation is not continual. What continues is not a situation which the subject has lived for 10 years but a situation itself living there. 
The perfect of proximal past: The perfect in many languages is used when a referred past situation is related to the present and it is temporally proximal. Temporally proximal is when the past situation happened in recent days.

\section{The aspect concept in Korean language}

The expression of tense or aspect in Korean language is not specified. But the aspect concept is presented through several limited prefinal endings. Among them, '-eot-'is widely used in various grammatical categories such as tense, aspect or viewpoint. There have been many arguments when it comes to a category and meaning function of '-eot'. Researchers say that it is a factor representing time-related meaning. But there are different opinions about whether it is considered as a tense or viewed as an aspect concept. For researchers insisting that '-eot' is a tense, '-eot' shows the meaning function of tense but it can also be interpreted as a meaning function of aspect according to the case.

The following is about the characteristics and limitation about the prefinal ending, '-eot' in Korean language. Its meaning function is viewed as a specific category like a tense or an aspect and the other meanings are how their meanings are simply interpreted. This is considered as an effort to verify a generality of the present related to '-eot'. In some arguments, like time aspect theory, its meaning function is different case by case, but its fundamental base is not definite. The studies where the meaning function of '-eot' is aspectual do not present a definite standard about correlation between aspectual traits and general system.

\subsection{The generalness of prefinal endings}

From '-eot' in Korean language, 'past, perfect, completion, result, situation, assurance or fact' are tense, aspectual and meaning. It is not easy to conclude a definition because these factors are included in it. The explanation can be easier if it has a common trait from a viewpoint of semantics. Thus I am going to find out semantically common things by analyzing prefinal endings.

Im, Hong Bin and Jang, So Won 1995:404) defined 'eot' as a 'confirmation of wholeness'. This means that the awareness of meaning can be differentiated depending on how a speaker is aware of the world, rather than simple tense meaning. This definition is considered as the same as Comrie (1976)'s point of view as I mentioned before. The interpretation as a tense expression of the past is possible based on the way a speaker is aware of the end of each situation. So, what is an exact concept of Comire's 'perfect' and Mr.Im's 'wholeness'? Also, is it okay that both of them is regarded as the same factors?

Briefly speaking, the wholeness is not mentioned in the concept of Comrie's perfect. His perfect aspect sees a situation as a whole, which means that a speaker looks at the situation from outside because the inner construction of time cannot be considered. In the wholeness of Comrie's perfect aspect, a situation is recognized as a whole unit. In contrast, Mr.Im's wholeness is defined as an end of cognition about the wholeness of an event. His wholeness is especially related with a way of awareness. Instead, in Comrie's aspectual category, there's a limit that inner time construction cannot be recognized because a situation is recognized as a unit. In contrast, time relation considered in the phase of situation development is not free, because Mr. Im's wholeness is the concept relating whether it is connected with a situation or not. 


\subsection{The completion of '-eot'}

The content of an utterance is a situation which exists in the real world is reflection. This moment of the situation is recognized through time and place of the real world. This time and place can generally be graphed as a time axis making a stream of time. But the utterance of letters which refers to a situation that exists in the real world may be a situation made by the speaker themselves. Or a situation may be simply an object of a speaker. If a speaker participated in a situation, the content of utterance is a situation where a speaker is reflected. So the speaker's viewpoint that exists on the axis of an ideal world is a reflected inner situation, where places on the time axis are the real world. In other words, the time axis of ideal world is reflected on the axis of real world.

\section{The time attribute of vocabulary and a situation}

The phrase like 'to lie on the table' is regarded as a lexical expression which does not include a right boundary line of time axis. On the other hand, the phrase like 'to put something on the table' includes the boundary line. But it is necessary to remind us that there are some cases that do not have the continuum in language. For example, it can be an instant such as the content of phrase like 'to arrive Spain' or a verb phrase likes 'to find out a solution'. In addition, the verb phrase presenting the situation like this cannot coexist with an adverb phrase presenting a continuum such as 'for two seconds' or 'since last year'.

Actually, a sense of differentiation between the real world and the language world is considerable. It seems that people want to focus on how a language reflects and is symbolized in the real world in the content of vocabulary, instead of telling how the real world is. Let's look at the following examples.

(2) a. Jane has slept in the guest bed.

b. Jane has closed the window.

In examples above, we have questions of how the difference can be explained between a state verb like 'has slept' and an action verb like 'has closed'. These are always optional descriptions when these verb phrases are used in a concrete utterance. The two situations include a time continuum distinctively measurable. If so, the vocabulary content like 'has slept' in (2a) does not contain a time boundary or it describes a situation not having a time boundary. On the other hand, it is impossible to figure out precisely what it means even though 'has close' from 2(b) contains a time boundary or it is interpreted being expressed by the boundary.

According to Klein (1994), one of many ways to a define time-based trait about a verb phrase and vocabulary aspect is observing a possible situation where a time-based trait of verb phrase can be applied .Another way is to require an inner language constraint. This is usually conducted by the following ways.

The modification of adverb phrase: The main issue is whether a used verb can combine with a specific adverb, such as 'He VP in two hours' or 'He VP for two hours' or not. Other than that, seeing if 'How long did it take to VP?' or a specific 'WH-' question can be used.

The modification by an aspect: The point is to check if a used vocabulary unit can be modified by an aspect. The most well-known is the '-ing' test. For example, the state verbs like 'contain, know, belong' do not allow progressive verbs.

presupposition and implication: When a sentence has an especially important form, checking if this sentence implies a presupposition or an implication. Examples of this are 'If 
someone is washing the car, then he has washed the car.' and 'if someone is living in London, then he has lived in London'. (Klein 1994: 71)

\section{Conclusion}

The attitudes toward a language can be defined by various points of view. Social characteristics, political and cultural factors have various impacts on it. In this journal, I have researched time-based expressions of English and Korean language which have different time and aspectual meanings. Though Korean language does not have specified time-based expressions compared English, the past prefinal ending '-eot' is a typical expression for replacing a present perfect 'have p.p.'. In the two languages from an aspectual point of view, inner traits and outer factors where a speaker sees situations are crucial factors when deciding an aspectual meaning of a sentence. A language form dominates the components, but a speaker's subjective, inner and outer situation can be an important indicator which shows an occurred event.

\section{References}

[1] R. J, Binnick, "Time and the Verb," Oxford, Blackwell

[2] C. Comrie, "Aspect, An Introduction to the study of Verbal Aspect and Related Problems," Cambridge University Press, vol.3, pp.18-19

[3] O. Dahl, "Tense and aspect systems," Oxford, Blackwell,

[4] N. I, Grech. "Prostrannaja russkaja grammatika," St Petersburg

[5] H.B. Lim and S.W. Jang, "Korean grammatical theory," The Air and Correspondence College, Seoul

[6] J. Lyons. "Dexis and subjectivity," Loquor, Ergo Sum 